OPEN ACCESS

Edited by:

Jing-Yan Han

Peking University, China

Reviewed by:

Alma Martelli,

Università degli Studi di Pisa, Italy

Suowen Xu,

University of Rochester, United States

*Correspondence:

Guo-Qing Zheng

gq_zheng@sohu.com

Yan Wang

wywzchina@sina.com

tThese authors have contributed equally to this work

Specialty section:

This article was submitted to Vascular Physiology,

a section of the journal

Frontiers in Physiology

Received: 20 October 2017 Accepted: 06 June 2018 Published: 03 July 2018

Citation:

Zheng Q, Zhu J-Z, Bao X-Y, Zhu P-C

Tong $Q$, Huang $Y-Y$, Zhang $Q-H$,

Zhang $K$-J, Zheng $G-Q$ and Wang $Y$ (2018) A Preclinical Systematic Review and Meta-Analysis of Astragaloside IV for Myocardial Ischemia/Reperfusion Injury. Front. Physiol. 9:795. doi: 10.3389/fphys.2018.00795

\section{A Preclinical Systematic Review and Meta-Analysis of Astragaloside IV for Myocardial Ischemia/Reperfusion Injury}

\author{
Qun Zheng ${ }^{1 t}$, Jia-Zhen Zhu ${ }^{1+}$, Xiao-Yi Bao ${ }^{1}$, Peng-Chong Zhu ${ }^{1}$, Qiang Tong ${ }^{1}$, \\ Yue-Yue Huang ${ }^{1}$, Qi-Hao Zhang ${ }^{1}$, Ke-Jian Zhang ${ }^{1}$, Guo-Qing Zheng ${ }^{2 *}$ and Yan Wang ${ }^{1 *}$ \\ ${ }^{1}$ Department of Cardiology, The Second Affiliated Hospital and Yuying Children's Hospital of Wenzhou Medical University, \\ Wenzhou, China, ${ }^{2}$ Department of Neurology, The Second Affiliated Hospital and Yuying Children's Hospital of Wenzhou \\ Medical University, Wenzhou, China
}

Astragaloside IV (AS-IV), the major pharmacological extract from Astragalus membranaceus Bunge, possesses a variety of biological activities in the cardiovascular systems. Here, we aimed to evaluate preclinical evidence and possible mechanism of AS-IV for animal models of myocardial ischemia/reperfusion (I/R) injury. Studies of AS-IV in animal models with myocardial I/R injury were identified from 6 databases from inception to May, 2018. The methodological quality was assessed by using CAMARADES 10-item checklist. All the data were analyzed using Rev-Man 5.3 software. As a result, 22 studies with 484 animals were identified. The quality score of studies ranged from 3 to 6 points. Meta-analyses showed AS-IV can significantly decrease the myocardial infarct size and left ventricular ejection fraction, and increase shortening fraction compared with control group $(P<0.01)$. Significant decreasing of cardiac enzymes and cardiac troponin and increasing of decline degree in ST-segment were reported in one study each $(P<0.05)$. Additionally, the possible mechanisms of AS-IV for myocardial I/R injury are promoting angiogenesis, improving the circulation, antioxidant, anti-inflammatory and anti-apoptosis. Thus, AS-IV is a potential cardioprotective candidate for further clinical trials of myocardial infarction.

\footnotetext{
Keywords: Astragaloside IV, myocardial ischemia/reperfusion injury, Astragalus membranaceus Bunge, preclinical systematic review, meta-analysis
}

\section{INTRODUCTION}

Acute myocardial infarction (AMI) was one of the leading causes of morbidity and mortality worldwide (Dariush et al., 2016). Acute interruption of coronary artery led to cardiomyocyte ischaemia and apoptosis (Luo et al., 2015). Invasive vascular reconstructions such as percutaneous coronary intervention and coronary artery bypass grafting can improve coronary perfusion (Richard, 2011), and thus they were widely adopted after weighing the risks of invasive diagnostics and the benefits in terms of diagnostic accuracy, risk stratification and assessment of the risks related to revascularization (Damman et al., 2015). Although treatment is usually directed at prompt restoration of flow in the occluded artery, reperfusion may trigger further injury beyond 
that induced by ischaemia alone (Maria et al., 2016). Such ischaemia/reperfusion (I/R) injury can markedly reduce the benefits of reperfusion therapies employed in myocardial infarction (MI) (Yellon and Hausenloy, 2007).

Astragaloside IV (AS-IV) (Figure 1) is one of the major and active components isolated from Astragalus membranaceus Bunge for tonifying $Q i$, and is a lanolin alcohol-shaped tetracyclic triterpenoid saponin with high polarity. Recent experimental studies (Ren et al., 2013; Li et al., 2017) demonstrated that AS-IV had pleiotropic anti-ischemic properties against focal cerebral ischemia/reperfusion injury, cardiovascular disease, pulmonary disease, liver fibrosis and diabetic nephropathy. AS-IV has multiple pharmacologic effects, including regulation of the calcium balance, antioxidative stress, anti-inflammatory, antiapoptosis antifibrotic, antidiabetes, immunoregulation, and cardioprotective effect via numerous signaling pathways (Schmidt et al., 2007; Ren et al., 2013; Li et al., 2017). In addition, systemic review of animal studies plays a critical role in drug development and the clarification of physiological and pathological mechanisms of clinical research (Rob et al., 2014). Thus, we conducted a preclinical systematic review to evaluate the effectiveness and the mechanisms of AS-IV for experimental MI.

\section{METHODS}

\section{Database and Literature Search Strategies}

Preferred Reporting Items for Systematic Review and MetaAnalyses (PRISMA)statement was followed (Stewart et al., 2015). Experimental studies assessing the effects of AS-IV in animal models of MI were identified from PubMed, EMBASE, Science Direct, Web of Science, wanfang data Information Site, Chinese National Knowledge Infrastructure (CNKI), and VIP information database by searching for all published articles from inception to May, 2018. The following key words were used: "astragaloside (MeSH Terms) OR astragaloside (Title/Abstract)" AND "myocardial infarction OR myocardial ischemia OR myocardial ischemia/reperfusion injury $\mathrm{OR}$ myocardial $\mathrm{I} / \mathrm{R}$

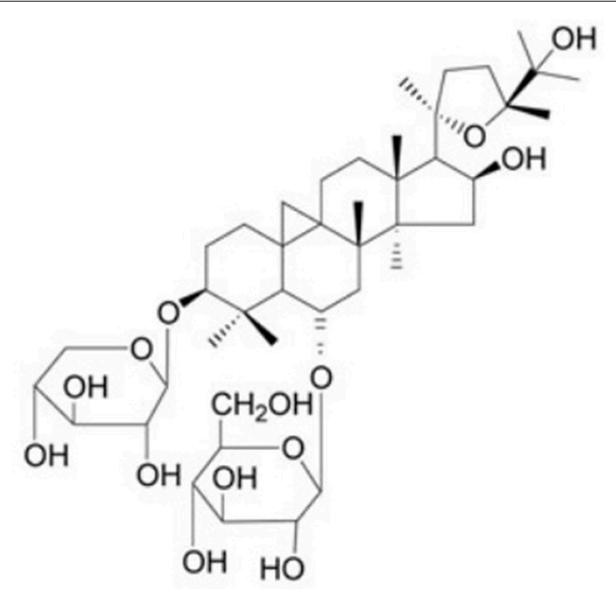

FIGURE 1 | Chemical structures of astragaloside IV. injury," Moreover, reference lists of potential articles were searched for additive studies.

\section{Eligibility Criteria}

We included studies of the effect of AS-IV in animal models of MI. To prevent bias, inclusion criteria were prespecified as follows: (1) experimental MI was induced by transient left anterior descending coronary artery (LAD) ligation or permanent LAD ligation or isoproterenol (ISO); (2) the treatment group was received AS-IV as monotherapy in any dose. Interventions for control group were isasteric and nonfunctional liquid (normal saline) or no treatment; (3) the primary outcome measures were MI size and/or left ventricular ejection fraction (LVEF) and/or shortening fraction (FS) and/or the level of ST-segment depression cardiac and/or enzymes and/or cardiac troponin $\mathrm{T}$ (cTnT) and/or cardiac troponin I (cTnI). The second outcome measures were mechanisms of AS-IV for myocardial I/R injury. Prespecified exclusion criteria were treatment with AS-IV conjunction with other compounds or AS-IV based prescriptions, non-myocardial ischemia model, no control group, duplicate publications, and no available data.

\section{Data Extraction}

Two independent authors extracted the following details from included studies: (1) publication year and the first author's name, model of MI (transient or permanent); (2) the characteristics of animals used including animal number, species, sex, weight, age, and any comorbidity; (3) model of myocardial I/R, and the anesthesia methods for model preparation; (4) the information of treatment group, including therapeutic drug dosage, method of administration, duration of treatment, and the same information of control group; (5) mean value and standard deviation of outcomes. If outcomes were performed at different time points, only the final test was included. If the experimental group of animals received various doses of the drug therapy, only the data of highest dose of the drug was included. If the data for metaanalysis were missing or only expressed graphically, we tried to contact the authors for further information, and where a response was not received, we measured data from the graphs using digital ruler software or exclude. For each comparison, we extracted data of mean value and standard deviation from each experimental and control group of every study.

\section{Quality Assessment}

We evaluated the methodological quality of the included studies using the collaborative Evidence-Based Complementary and Alternative Medicine approach to meta-analysis and review of animal data in experimental infarction (CAMARADES) 10-item quality checklist (Malcolm et al., 2004) with minor modification (Yu L. J. et al., 2017). One point was awarded for each of (1) publication in a peer-reviewed journal; (2) statement of temperature control; (3) random allocation to groups; (4) allocation concealment; (5) blinded assessment of outcome; (6) use of anesthetic without significant intrinsic cardioprotective activity; (7) appropriate animal model (aged, diabetic, or hypertensive); (8) sample size calculation; (9) compliance with animal welfare regulations; (10) statement of potential conflict of interests. 


\section{Statistical Analysis}

All CI were considered as continuous data, and then an estimate of the combined effect sizes utilizing mean difference (MD) or standard mean difference (SMD) with the random effects model was given. In the present meta-analysis, the results using the random effects model were presented because heterogeneity between multistudies has to be taken into account. $I^{2}$ statistic was used to assess heterogeneity. The significance of differences among groups was assessed by partitioning heterogeneity and by using the $X^{2}$ distribution with degrees of freedom (df), where equals the number of groups. Probability values 0.05 were considered significant. All analyses were performed with Revman version 5.3 by the Cochrane Collaboration.

\section{RESULTS}

\section{Study Inclusion}

We identified 1,280 potentially relevant articles, of which 1,045 were reduplicated and irrelevant articles. Through screening titles and abstracts, 48 papers were excluded with at least one of following reasons: (1) clinical trial; (2) case report; (3) review article. By reading the fulltext of the remaining 87 articles, 65 articles were excluded because of at least one of the following reasons: (1) not full text; (2) not AS-IV intervention; (3) no available data; (4) compared with traditional Chinese medicine; (5) no MI/R model; (6) no control group; (7) no available data. Ultimately, 22 eligible articles (Zhang et al., 2005, 2014; Zhao et al., 2009; Guan et al., 2010; Liu et al., 2010; Wang et al., 2012, 2018; Cui et al., 2013; Gong and Sun, 2013; Tu et al., 2013; He et al., 2014; Liu and Yi, 2014; Qu et al., 2014; Huang et al., 2015; Lu et al., 2015; Ma and Wang, 2015; Sun et al., 2015; Yu et al., 2015; Cheng et al., 2016; Li and Yang, 2016; Li et al., 2016; Yu J. et al., 2017) were identified (Figure 2).

\section{Study Characteristics}

A total of 484 animals were included in the 22 studies, of which 234 were in the experimental group and 230 were in the control group. Eight studies (Zhang et al., 2005; Wang et al., 2012; Gong and Sun, 2013; Tu et al., 2013; Lu et al., 2015; Yu et al., 2015; Cheng et al., 2016; Yu J. et al., 2017) were published in English and 14 studies (Zhao et al., 2009; Guan et al., 2010; Liu et al., 2010; Cui et al., 2013; He et al., 2014; Liu and Yi, 2014; Qu et al., 2014; Zhang et al., 2014; Huang et al., 2015; Ma and Wang, 2015; Sun et al., 2015; Li and Yang, 2016; Li et al., 2016; Wang et al.,

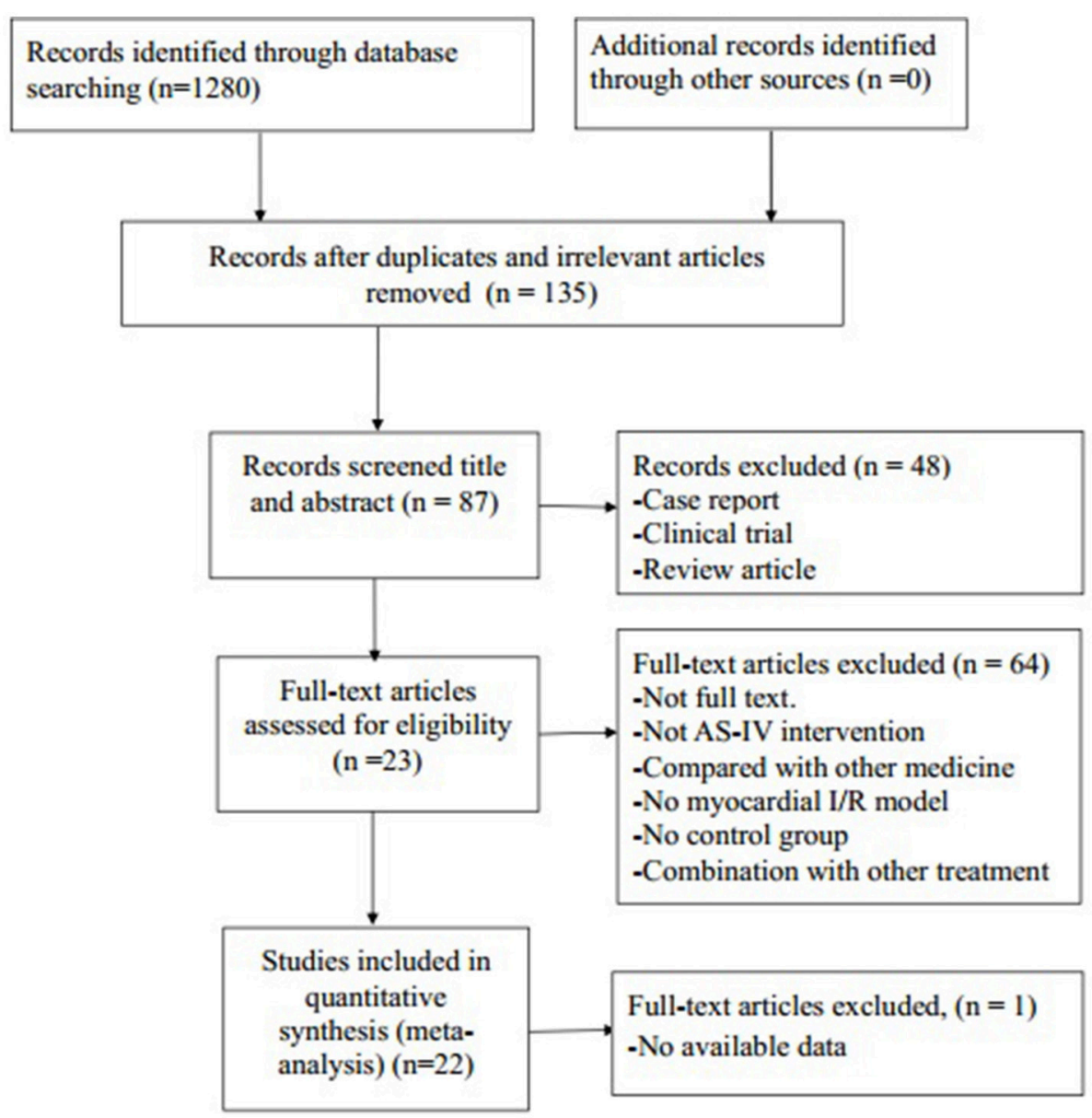

FIGURE 2 | Summary of the process for identifying candidate studies. 
2018) were published in Chinese between 2005 and 2018. Twelve studies (Cui et al., 2013; Gong and Sun, 2013; Tu et al., 2013; Liu and Yi, 2014; Qu et al., 2014; Zhang et al., 2014; Huang et al., 2015; Lu et al., 2015; Ma and Wang, 2015; Sun et al., 2015; Cheng et al., 2016; Wang et al., 2018) used Sprague Dawley rats; 6 studies (Zhao et al., 2009; Guan et al., 2010; Wang et al., 2012; He et al., 2014; Yu et al., 2015; Yu J. et al., 2017) used Wistar rats; 1 study (Li et al., 2016) used unknown breed rats; 1 study (Li et al., 2016) used C57/BL6 mice; 1 study (Liu et al., 2010) used Beagle dogs and 1 study (Zhang et al., 2005) used Mongrel dogs. Eighteen studies (Zhang et al., 2005, 2014; Zhao et al., 2009; Guan et al., 2010; Wang et al., 2012, 2018; Cui et al., 2013; Tu et al., 2013; He et al., 2014; Liu and Yi, 2014; Qu et al., 2014; Huang et al., 2015; Lu et al., 2015; Ma and Wang, 2015; Yu et al., 2015; Cheng et al., 2016; Li and Yang, 2016; Yu J. et al., 2017) used male animals, two studies (Liu et al., 2010; Cui et al., 2013) used both female and male rats, and two studies (Sun et al., 2015; Li et al., 2016) did not mention gender of animals. All studies reported that myocardial $\mathrm{I} / \mathrm{R}$ models were produced by ligation of the LAD. To induce anesthesia, 3 studies (Wang et al., 2012; Gong and Sun, 2013; Ma and Wang, 2015) used chloral hydrate; 13 studies (Zhang et al., 2005, 2014; Cui et al., 2013; Tu et al., 2013; He et al., 2014; Liu and Yi, 2014; Qu et al., 2014; Huang et al., 2015; Yu et al., 2015; Cheng et al., 2016; Li and Yang, 2016; Li et al., 2016; Yu J. et al., 2017) used pentobarbital sodium; 3 studies (Guan et al., 2010; Lu et al., 2015; Wang et al., 2018) used urethane; and anesthetic was not mentioned in remaining 3 studies (Zhao et al., 2009; Huang et al., 2015; Sun et al., 2015). Among the dose use of AS-IV, 1

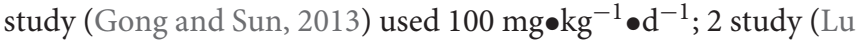
et al., 2015; Wang et al., 2018) used $80 \mathrm{mg} \bullet \mathrm{kg}^{-1} \bullet \mathrm{d}^{-1}$; 1 study (Cheng et al., 2016) used 50 mg•kg ${ }^{-1} \bullet d^{-1} ; 1$ study (Wang et al., 2012) used $40 \mathrm{mg} \mathrm{kg}^{-1} \bullet \mathrm{d}^{-1}$; 10 studies (Cui et al., 2013; Tu et al., 2013; He et al., 2014; Zhang et al., 2014; Ma and Wang, 2015; Sun et al., 2015; Yu et al., 2015; Li and Yang, 2016; Yu J. et al., 2017) utilized $10 \mathrm{mg} \bullet \mathrm{kg}^{-1} \bullet \mathrm{d}^{-1} ; 1$ study (Guan et al., 2010) used 5 mg•kg-1 $\bullet \mathrm{d}^{-1}$; 2 studies (Qu et al., 2014; Huang et al., 2015) adopted $4 \mathrm{mg} \bullet \mathrm{kg}^{-1} \bullet \mathrm{d}^{-1}$; 1 study (Li et al., 2016) used $2 \mathrm{mg} \mathrm{kg}^{-1} \bullet \mathrm{d}^{-1}$; 1 study (Zhang et al., 2005) used 1.5 $\mathrm{mg} \bullet \mathrm{kg}^{-1} \bullet \mathrm{d}^{-1} ; 1$ study (Liu and Yi, 2014) used $50 \mathrm{uM} / \mathrm{kg}$; the remaining 2 studies (Zhao et al., 2009; Liu et al., 2010) used 1 mg•kg-1 ${ }^{-1}$ Ten studies (Zhang et al., 2005, 2014; Tu et al., 2013; He et al., 2014; Liu and Yi, 2014; Lu et al., 2015; Cheng et al., 2016; Li et al., 2016; Yu J. et al., 2017; Wang et al., 2018) utilized MI size as outcome measure, and myocardial cell apoptosis rate in 5 studies (Zhao et al., 2009; Liu and Yi, 2014; Lu et al., 2015; Ma and Wang, 2015; Sun et al., 2015). The level of ST-segment depression was reported in 1 study (Liu et al., 2010), the LVEF in 5 studies (Wang et al., 2012; Zhang et al., 2014; Cheng et al., 2016; Li and Yang, 2016; Li et al., 2016) and FS in 6 studies (Zhao et al., 2009; Wang et al., 2012; Zhang et al., 2014; Cheng et al., 2016; Li and Yang, 2016; Li et al., 2016). Lactate dehydrogenase (LDH) was reported in 2 studies (Guan et al., 2010; Qu et al., 2014) creatine kinase (CK) in 1 study (Qu et al., 2014), cTnT in 1 study (Tu et al., 2013); cTnI in 1 study (Wang et al., 2018), superoxide dismutase (SOD) in 1 study (Guan et al., 2010), malondialdehyde (MDA) in 1 study (Guan et al., 2010), hypoxia-inducible factor 1- $\alpha$ (HIF1 $\alpha$ ) 1 study (Yu J. et al., 2017), caspase-3 in 3 studies (Liu and
Yi, 2014; Lu et al., 2015; Ma and Wang, 2015), calcium-sensing receptor vascular in 1 study (Wang et al., 2018), endothelium growth factor (VEGF) in 2 studies (Yu et al., 2015; Li et al., 2016) tumor necrosis factor- $\alpha$ (TNF- $\alpha$ ) in 1 study (Lu et al., 2015), nuclear factor $\kappa \mathrm{B}(\mathrm{NF}-\kappa \mathrm{B})$ in 3 study (Tu et al., 2013; Lu et al., 2015; Cheng et al., 2016), NO in 1 study (Guan et al., 2010), coronary blood flow (CBF) in 2 studies (Zhang et al., 2005; Liu et al., 2010), microvessel density (MVD) in 3 studies (Yu et al., 2015; Li and Yang, 2016; Li et al., 2016), and Bax and Bcl-2 in 7 studies (Zhao et al., 2009; Tu et al., 2013; Liu and Yi, 2014; Lu et al., 2015; Ma and Wang, 2015; Cheng et al., 2016; Wang et al., 2018). The characteristics of the 22 included studies were summarized in detail in Table $\mathbf{1 .}$

\section{Study Quality}

All the included records were peer reviewed publications and all animals were allocated randomly to treatment group and control group; however, no study reported a sample size calculation, blinded induction of model and blinding their assessment of outcome. Nine studies (Zhao et al., 2009; Wang et al., 2012; Tu et al., 2013; Lu et al., 2015; Yu et al., 2015; Cheng et al., 2016; Li and Yang, 2016; Li et al., 2016; Yu J. et al., 2017) reported control of temperature. Nine studies (Zhang et al., 2005, 2014; Zhao et al., 2009; Wang et al., 2012; Tu et al., 2013; Lu et al., 2015; Yu et al., 2015; Cheng et al., 2016; Yu J. et al., 2017) reported a compliance with animal welfare regulations, and 6 studies (Zhao et al., 2009; Wang et al., 2012; Lu et al., 2015; Cheng et al., 2016; Li and Yang, 2016; Yu J. et al., 2017) mentioned a statement of potential conflict of interests. Five studies (Liu et al., 2010; Qu et al., 2014; Huang et al., 2015; Sun et al., 2015; Yu et al., 2015) described appropriate animal models (aged, diabetic, or hypertensive). All studies except two studies (Zhao et al., 2009; Sun et al., 2015) used an anesthetic without intrinsic cardioprotective properties. To summarize, the quality score of included studies ranges from 3 to 6. Of which, 10 studies (Zhang et al., 2005; Guan et al., 2010; Cui et al., 2013; Gong and Sun, 2013; He et al., 2014; Liu and Yi, 2014; Huang et al., 2015; Ma and Wang, 2015; Sun et al., 2015; Wang et al., 2018) got 3 points; 5 studies (Liu et al., 2010; Tu et al., 2013; Qu et al., 2014; Zhang et al., 2014; Li et al., 2016) got 4 points; 3 studies (Zhao et al., 2009; Yu et al., 2015; Li and Yang, 2016) got 5 points; and 4 studies (Wang et al., 2012; Lu et al., 2015; Cheng et al., 2016; Yu J. et al., 2017) got 6 points. The methodological quality of each study was summarized in Table 2.

\section{Effectiveness \\ MI Size}

Ten studies utilized MI size (Zhang et al., 2005, 2014; Tu et al., 2013; He et al., 2014; Liu and Yi, 2014; Lu et al., 2015; Cheng et al., 2016; Li et al., 2016; Yu J. et al., 2017; Wang et al., 2018) as outcome measure. All of them showed significant effect of AS-IV for decreasing the MI size $(P<0.05)$. However, 1 study (Liu and Yi, 2014) that MI experimental model was induced by blocking LAD for 90 vs. 30 min in other studies; 1 study (Tu et al., 2013) showed that the data of MI size is inconsistent between figure and form; 1 study (Yu J. et al., 2017) only observe the gross cardiac morphology without measuring data. Meta-analysis of 7 studies (Zhang et al., 2005, 2014; He et al., 2014; Lu et al., 2015; 


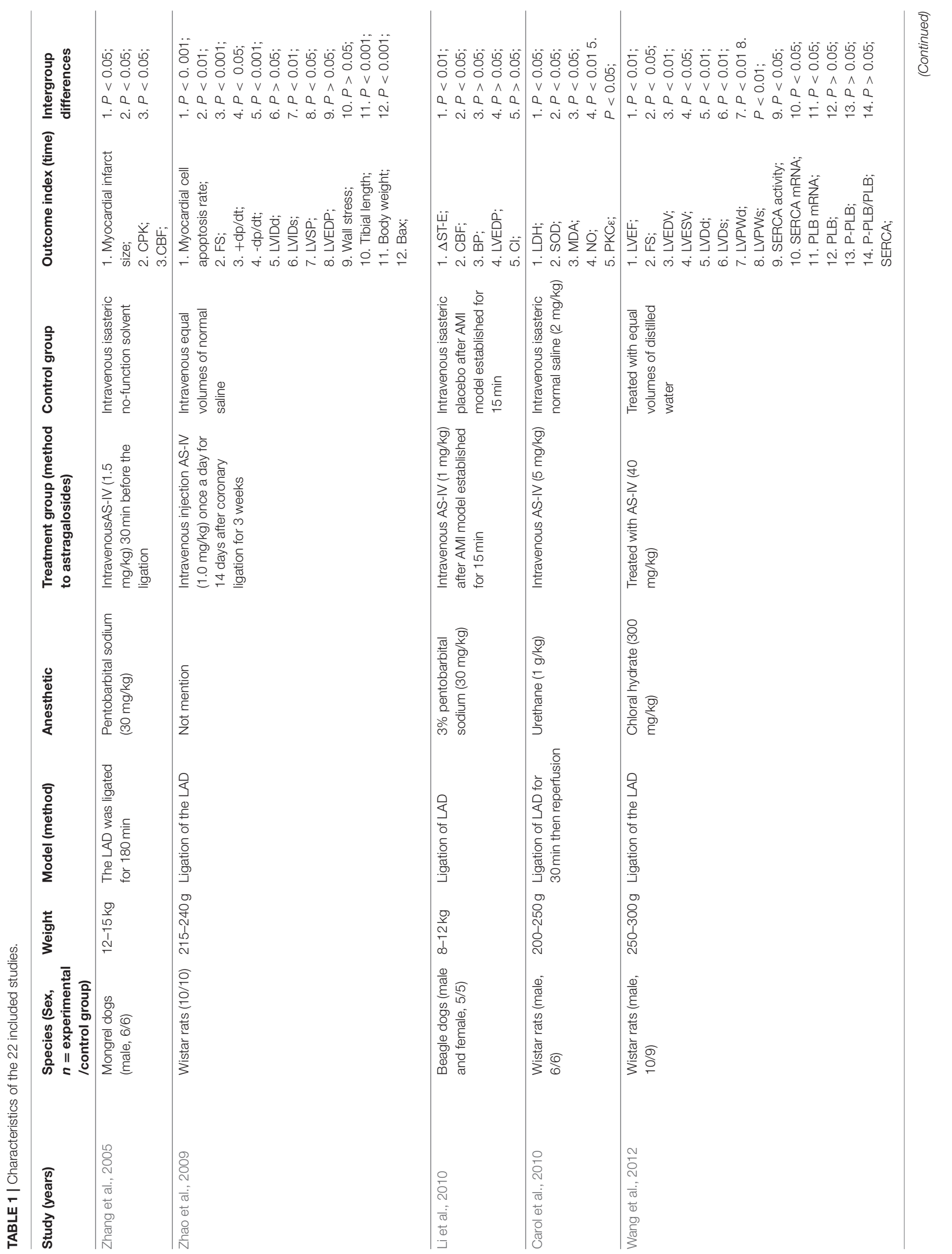




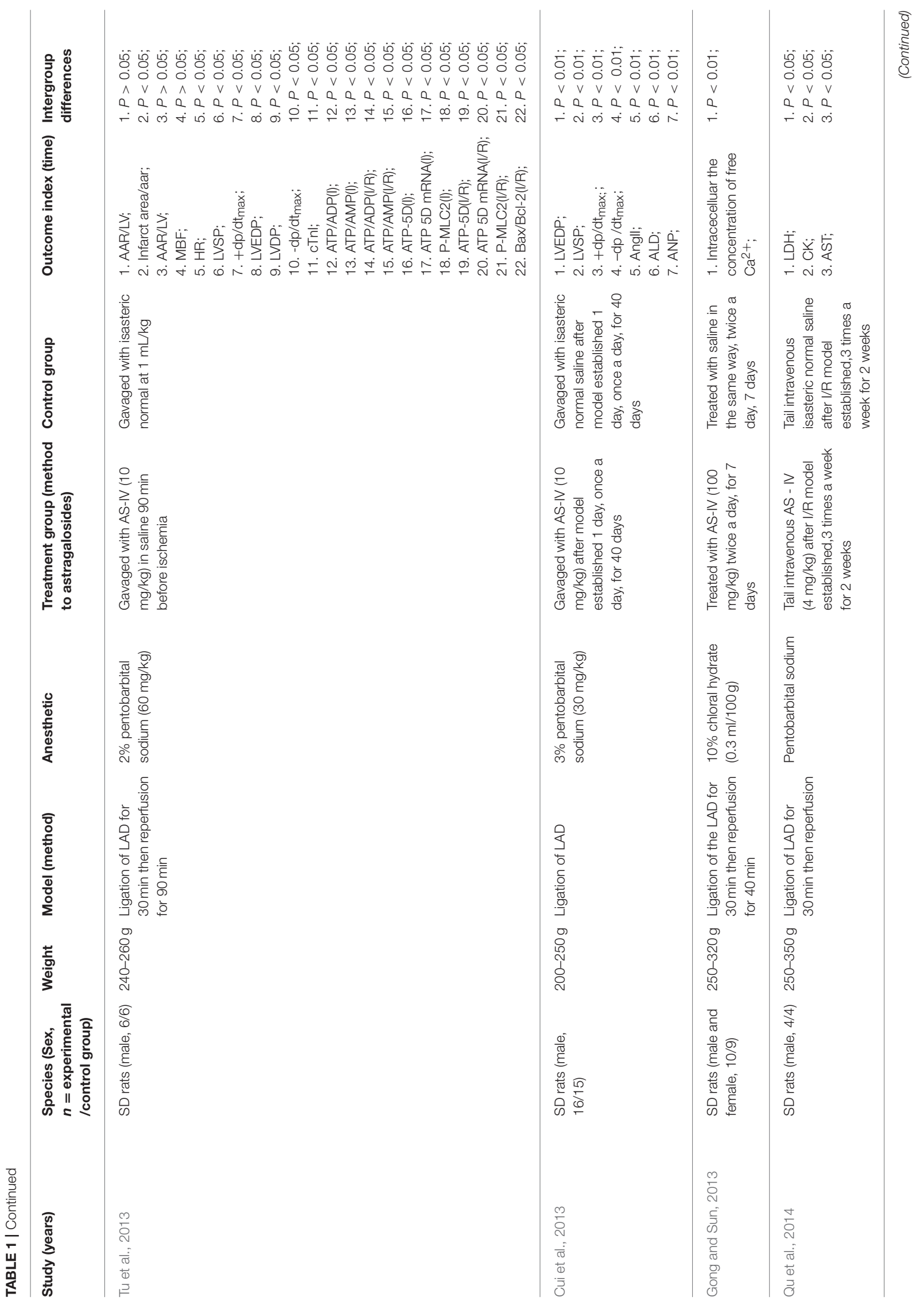




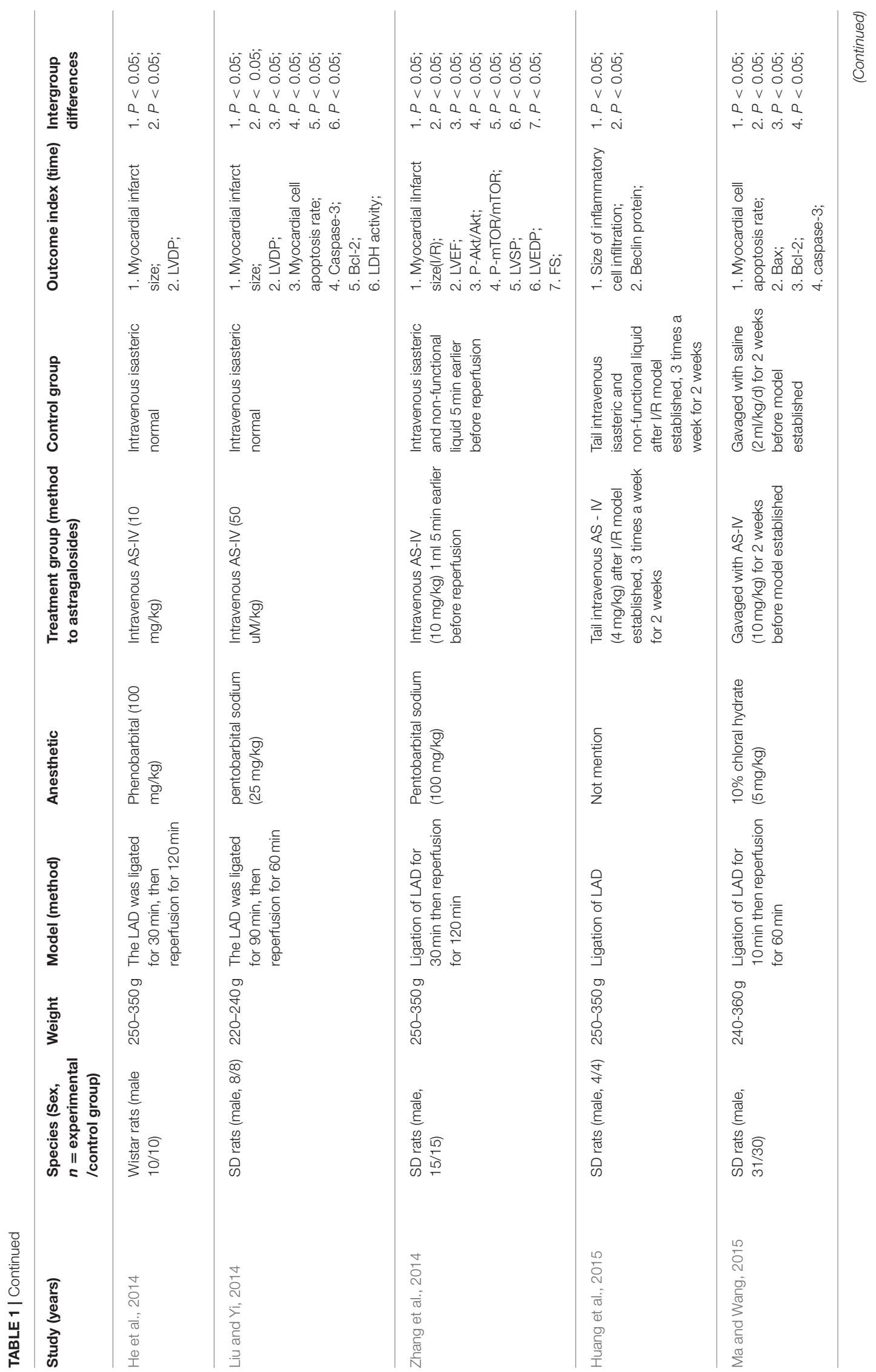




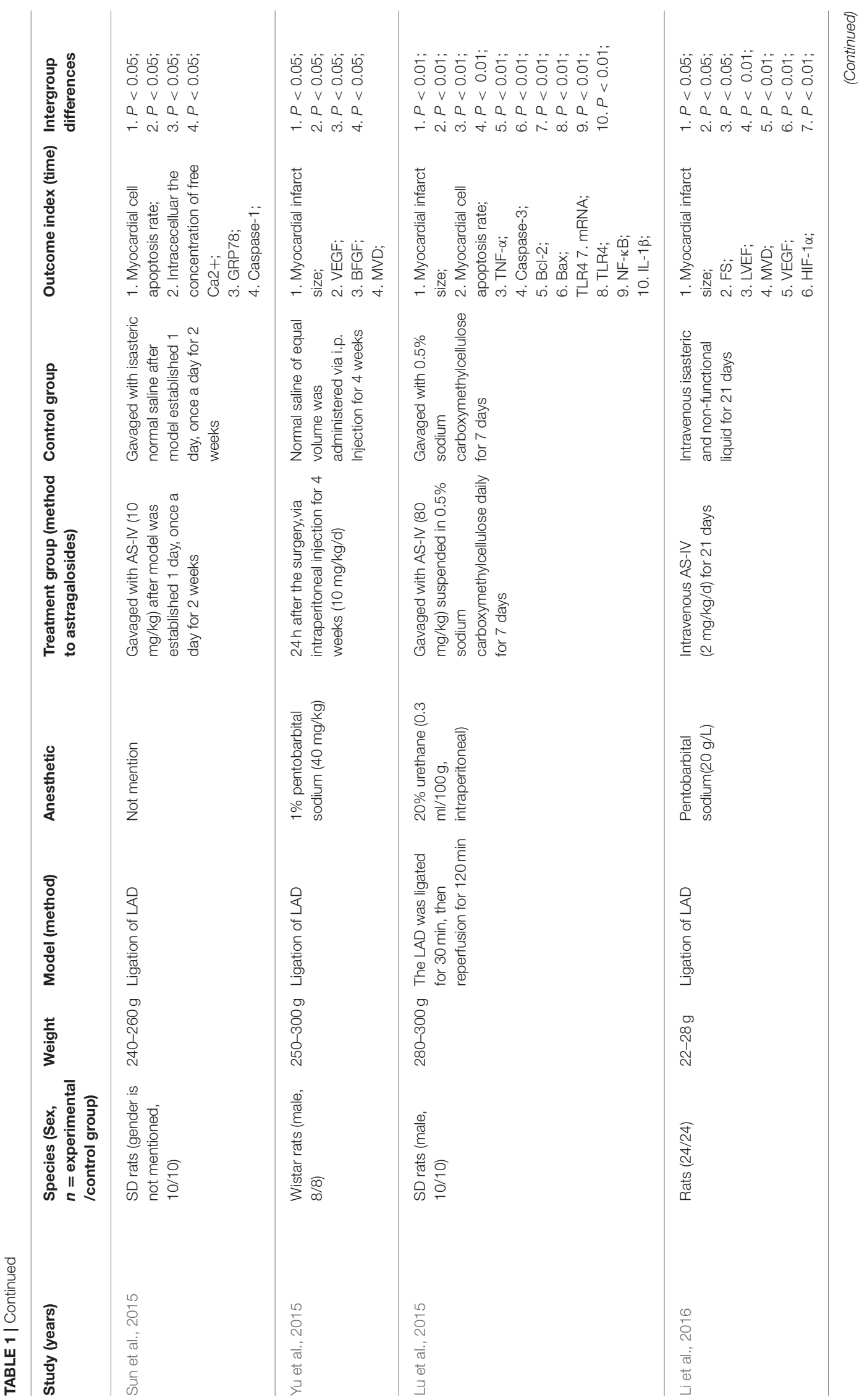




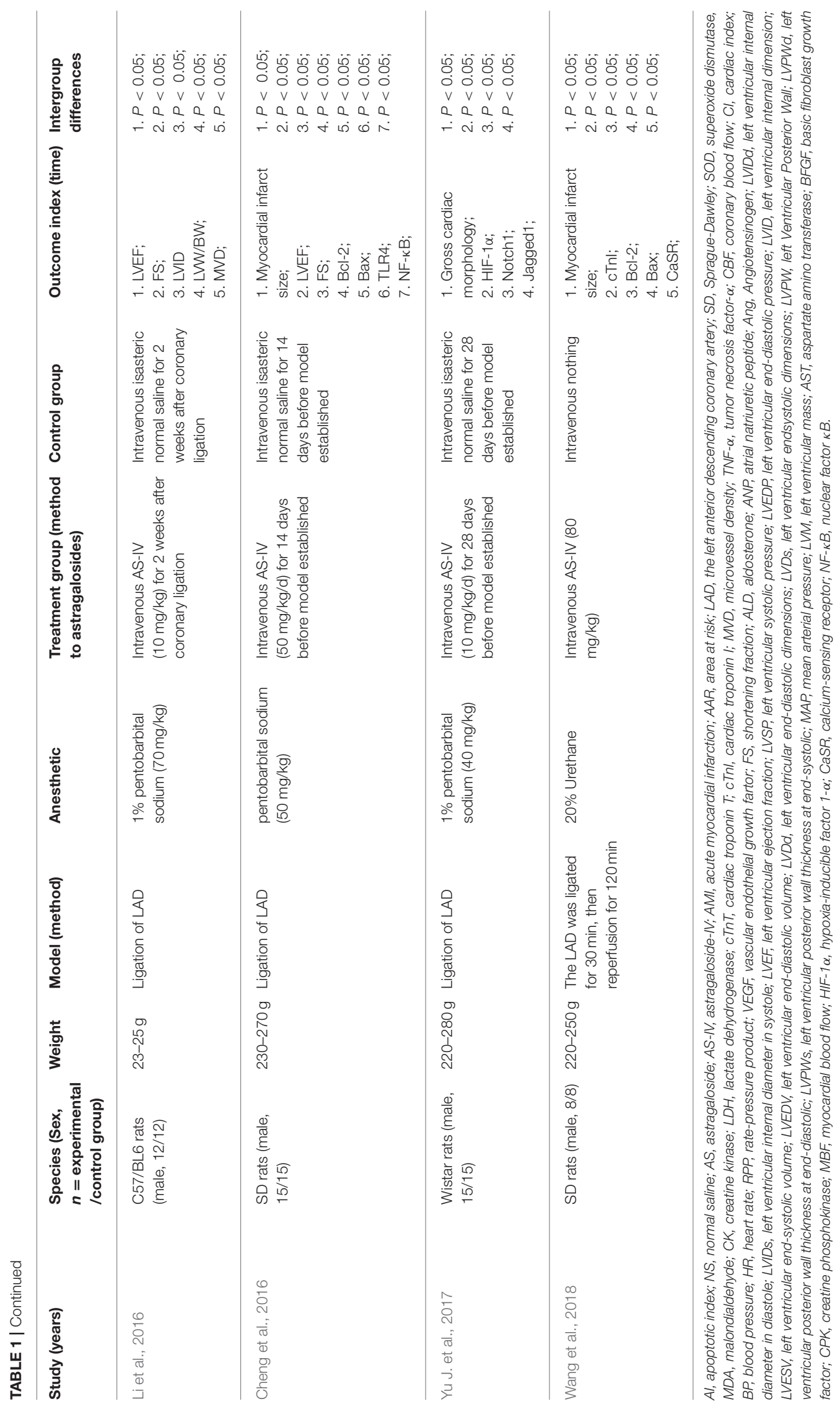


TABLE 2 | Risk of bias of the included studies.

\begin{tabular}{|c|c|c|c|c|c|c|c|c|c|c|c|}
\hline Study & A & B & C & D & $\mathbf{E}$ & $\mathbf{F}$ & G & H & I & $\mathbf{J}$ & Total \\
\hline Zhang et al., 2014 & $\sqrt{ }$ & & $\sqrt{ }$ & & & $\sqrt{ }$ & & & $\sqrt{ }$ & & 4 \\
\hline Zhang et al., 2005 & $\sqrt{ }$ & & & & & $\sqrt{ }$ & & & $\sqrt{ }$ & & 3 \\
\hline Zhao et al., 2009 & $\sqrt{ }$ & $\sqrt{ }$ & $\sqrt{ }$ & & & & & & $\sqrt{ }$ & $\sqrt{ }$ & 5 \\
\hline Liu et al., 2010 & $\sqrt{ }$ & & $\sqrt{ }$ & & & $\sqrt{ }$ & $\sqrt{ }$ & & & & 4 \\
\hline Guan et al., 2010 & $\sqrt{ }$ & & $\sqrt{ }$ & & & $\sqrt{ }$ & & & & & 3 \\
\hline Wang et al., 2012 & $\sqrt{ }$ & $\sqrt{ }$ & $\sqrt{ }$ & & & $\sqrt{ }$ & & & $\sqrt{ }$ & $\sqrt{ }$ & 6 \\
\hline Cui et al., 2013 & $\sqrt{ }$ & & $\sqrt{ }$ & & & $\sqrt{ }$ & & & & & 3 \\
\hline Tu et al., 2013 & $\sqrt{ }$ & $\sqrt{ }$ & & & & $\sqrt{ }$ & & & $\sqrt{ }$ & & 4 \\
\hline Gong and Sun, 2013 & $\sqrt{ }$ & & $\sqrt{ }$ & & & $\sqrt{ }$ & & & & & 3 \\
\hline Qu et al., 2014 & $\sqrt{ }$ & & $\sqrt{ }$ & & & $\sqrt{ }$ & $\sqrt{ }$ & & & & 4 \\
\hline He et al., 2014 & $\sqrt{ }$ & & $\sqrt{ }$ & & & $\sqrt{ }$ & & & & & 3 \\
\hline Liu and Yi, 2014 & $\sqrt{ }$ & & $\sqrt{ }$ & & & $\sqrt{ }$ & & & & & 3 \\
\hline Huang et al., 2015 & $\sqrt{ }$ & & $\sqrt{ }$ & & & & $\sqrt{ }$ & & & & 3 \\
\hline Ma and Wang, 2015 & $\sqrt{ }$ & & $\sqrt{ }$ & & & $\sqrt{ }$ & & & & & 3 \\
\hline Sun et al., 2015 & $\sqrt{ }$ & & $\sqrt{ }$ & & & & $\sqrt{ }$ & & & & 3 \\
\hline Yu et al., 2015 & $\sqrt{ }$ & $\sqrt{ }$ & & & & $\sqrt{ }$ & $\sqrt{ }$ & & $\sqrt{ }$ & & 5 \\
\hline Lu et al., 2015 & $\sqrt{ }$ & $\sqrt{ }$ & $\sqrt{ }$ & & & $\sqrt{ }$ & & & $\sqrt{ }$ & $\sqrt{ }$ & 6 \\
\hline Li and Yang, 2016 & $\sqrt{ }$ & $\sqrt{ }$ & $\sqrt{ }$ & & & $\sqrt{ }$ & & & & & 4 \\
\hline Li and Yang, 2016 & $\sqrt{ }$ & $\sqrt{ }$ & $\sqrt{ }$ & & & $\sqrt{ }$ & & & & $\sqrt{ }$ & 5 \\
\hline Cheng et al., 2016 & $\sqrt{ }$ & $\sqrt{ }$ & $\sqrt{ }$ & & & $\sqrt{ }$ & & & $\sqrt{ }$ & $\sqrt{ }$ & 6 \\
\hline Yu J. et al., 2017 & $\sqrt{ }$ & $\sqrt{ }$ & $\sqrt{ }$ & & & $\sqrt{ }$ & & & $\sqrt{ }$ & $\sqrt{ }$ & 6 \\
\hline Wang et al., 2018 & $\sqrt{ }$ & & $\sqrt{ }$ & & & $\sqrt{ }$ & & & & & 3 \\
\hline
\end{tabular}

Studies fulfiling the criteria of: $A$, peer reviewed publication; B, control of temperature; $C$, random allocation to treatment or control; $D$, blinded induction of model; $E$, blinded assessment of outcome; $F$, use of anesthetic without significant intrinsic cardioprotective activity; G, appropriate animal model (aged, diabetic, or hypertensive); H, sample size calculation; I, compliance with animal welfare regulations; J, statement of potential conflict of interest.

Cheng et al., 2016; Li et al., 2016; Wang et al., 2018) showed significant effect of AS-IV for decreasing the MI size compared with control group $[n=63$, SMD $-2.58,95 \%$ CI $[-3.10$ to -2.06], $P<0.01$; heterogeneity: $\chi^{2}=11.81, d f=6(P=0.07)$; $\left.I^{2}=49 \%\right]$ (Figure 3).

\section{LVEF}

Meta-analysis 5 studies (Wang et al., 2012; Zhang et al., 2014; Cheng et al., 2016; Li and Yang, 2016; Li et al., 2016) showed significant effects of AS-IV for improving LVEF compared with control group $[n=69$, SMD 3.23, 95\% CI: 2.17-4.30, $P<0.00001$; heterogeneity: $\chi^{2}=14.62, d f=4(P=0.006)$, $I^{2}=73 \%$ ]. After removing 1 study (Wang et al., 2012), the animal was treated with AS-IV at 5 weeks after MI other than immediately after establishing the MI model in other studies. Meta-analysis 3 studies (Zhang et al., 2014; Cheng et al., 2016; Li and Yang, 2016; Li et al., 2016) showed significant effects of AS-IV for improving LVEF compared with control group $[n=59$, SMD 3.63, 95\% CI: 2.96-4.31, $P<0.0001$; heterogeneity: $\chi^{2}=3.41$, $d f=3(P=0.33), I^{2}=12 \%$ ] (Figure 4). The study (Wang et al., 2012) showed significant effect of AS-IV for improving the LVEF $(P<0.05)$.

\section{FS}

Meta-analysis of 6 studies (Zhao et al., 2009; Wang et al., 2012; Zhang et al., 2014; Cheng et al., 2016; Li and Yang, 2016; Li et al., 2016) showed significant effects of AS-IV for improving FS compared with control group $[n=57, \mathrm{MD} 10.28,95 \% \mathrm{CI}$ : 6.78-13.77, $P<0.0001$; heterogeneity: $\chi^{2}=41.25, d f=5$ $(P<0.0001), I^{2}=88 \%$ ]. After sensitivity analyses, we removed 1 study (Li and Yang, 2016) that was more than 30\% died rate during experiment. Meta-analysis of 4 studies (Zhao et al., 2009; Wang et al., 2012; Zhang et al., 2014; Cheng et al., 2016; Li et al., 2016) showed significant effects of AS-IV for improving FS compared with control group $[n=49$, MD 11.60, 95\% CI: $10.32-$ 12.88, $P<0.0001$; heterogeneity: $\chi^{2}=4.14, d f=4(P=0.39)$, $\left.I^{2}=3 \%\right]$ (Figure 5).

\section{The Level of ST-Segment Depression}

One study (Liu et al., 2010) reported that AS-IV can improve the ST-segment depression compared with control $(P<0.05)$.

\section{Cardiac Enzymes and/or Cardiac Troponin}

Meta-analysis of 2 studies (Guan et al., 2010; Qu et al., 2014) showed significant effect of AS-IV for decreasing the LDH compared with control group $[n=12, \mathrm{SMD}-1.92,95 \% \mathrm{CI}$ [ -2.99 to -0.86$], P<0.01$; heterogeneity: $\chi^{2}=1.57, d f=1$ $(P=0.21) ; I^{2}=36 \%$, (Figure 6). CK, cTnT, and cTnI were reported in one study respectively (Tu et al., 2013; Qu et al., 2014; Wang et al., 2018), they showed that AS-IV had significant effects for reducing $\mathrm{CK}, \mathrm{cTnT}$ and $\mathrm{cTnI}$ compared with control group $(P<0.05)$.

\section{Cardioprotective Mechanisms}

Compared with controls, meta-analysis of 2 studies (Yu et al., 2015; Li et al., 2016) showed that AS-IV significantly increasing VEGF $[n=32$, SMD 2.23, 95\% CI [1.31-3.16], $P<0.01$; heterogeneity: $\left.\chi^{2}=1.77, d f=1(P=0.18) ; I^{2}=44 \%\right]$, (Figure 7A); 3 studies (Yu et al., 2015; Li and Yang, 2016; Li et al., 2016) for increasing MVD $[n=38$, SMD 2.22, 95\% CI [1.622.82], $P<0.01$; heterogeneity: $\chi^{2}=1.59, d f=2(P=0.45)$; $I^{2}=0 \%$, (Figure 7B); 2 studies (Lu et al., 2015; Sun et al., 2015) for reducing myocardial cell apoptosis rate after sensitivity analyses, $[n=20, \mathrm{MD}-13.78,95 \% \mathrm{CI}[-14.63$ to -12.93$]$, $P<0.01$; heterogeneity: $\left.\chi^{2}=0.69, d f=1(P=0.41) ; I^{2}=0 \%\right]$, (Figure 7C); 2 studies (Zhang et al., 2005; Liu et al., 2010) showed that AS-IV significantly increasing CBF $[n=11$, SMD 4.40, 95\% CI [2.84-5.96], $P<0.01$; heterogeneity: $\chi^{2}=0.92, d f=1$ $\left.(\mathrm{P}=0.34) ; I^{2}=0 \%\right]$, (Figure 7D); 1 study (Lu et al., 2015) for decreasing TNF- $\alpha(P<0.05)$; 3 study (Tu et al., 2013; Lu et al., 2015; Cheng et al., 2016) for decreasing NF-кB; 1 study (Yu J. et al., 2017) for increasing HIF-1 $\alpha$; 1 study (Guan et al., 2010) for decreasing SOD, MDA and NO $(P<0.05)$. In addition, 7 studies (Zhao et al., 2009; Tu et al., 2013; Liu and Yi, 2014; Lu et al., 2015; Ma and Wang, 2015; Cheng et al., 2016; Wang et al., 2018) which utilized Bax and Bcl-2 as outcome measure failed for pooling analysis, however they all reported that ASIV significantly increasing Bcl-2 and decreasing $\operatorname{Bax}(P<0.05)$. We summarized a schematic representation of cardioprotective mechanism of AS-IV for myocardial I/R injury (Figure 8). 


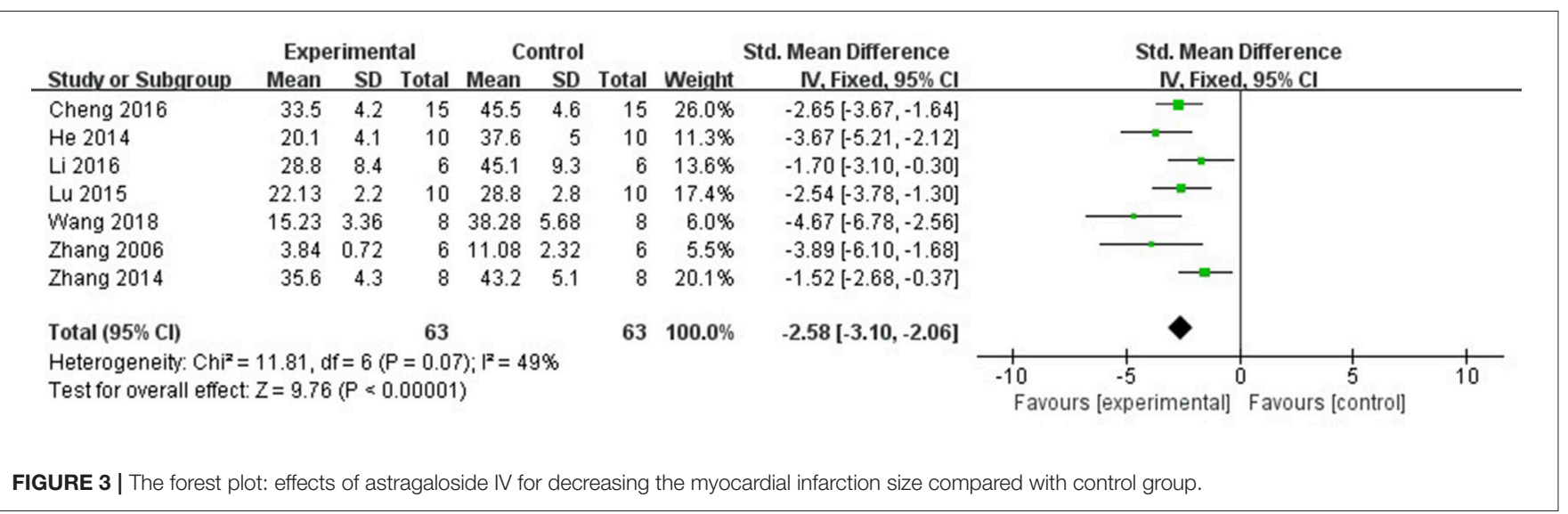

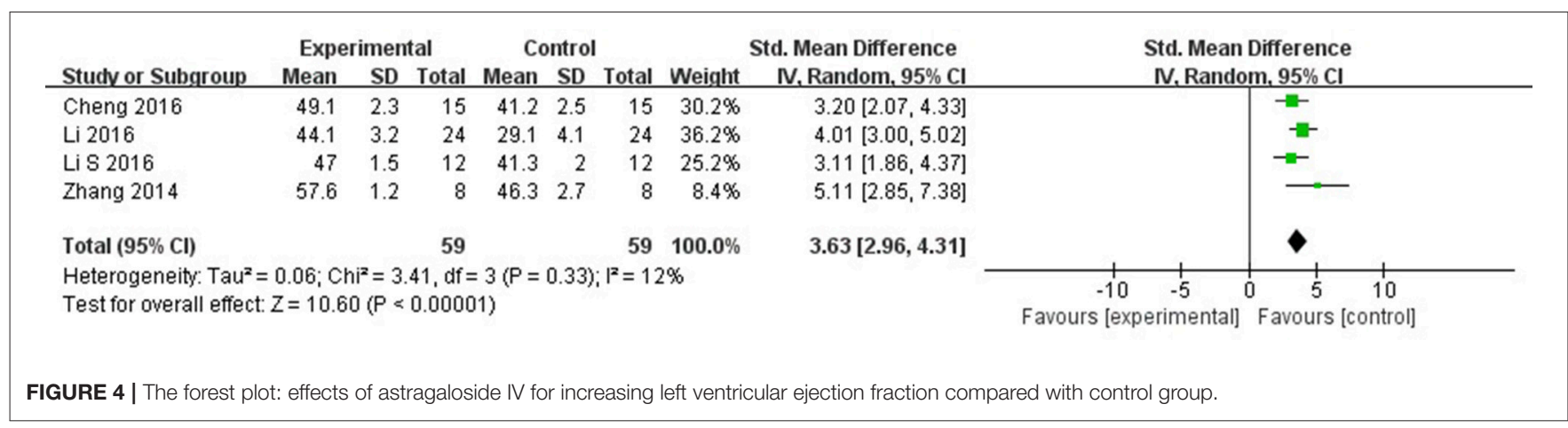

\begin{tabular}{|c|c|c|c|c|c|c|c|c|c|c|}
\hline \multirow[b]{2}{*}{ Study or Subgroup } & \multicolumn{3}{|c|}{ Experimental } & \multicolumn{2}{|c|}{ Control } & \multirow{2}{*}{ Total } & \multirow[b]{2}{*}{ Weight } & \multirow{2}{*}{$\begin{array}{l}\text { Mean Difference } \\
\text { IV, Random, } 95 \% \mathrm{Cl}\end{array}$} & \multirow{2}{*}{\multicolumn{2}{|c|}{$\begin{array}{c}\text { Mean Difference } \\
\text { IV, Random, } 95 \% \mathrm{Cl}\end{array}$}} \\
\hline & Mean & SD & Total & Mean & SD & & & & & \\
\hline Cheng 2016 & 28.8 & 2.2 & 15 & 17.3 & 2.5 & 15 & $51.7 \%$ & $11.50[9.81,13.19]$ & & 들 \\
\hline Li 2016 & 44.1 & 3.2 & 6 & 29.1 & 4.1 & 6 & $9.3 \%$ & $15.00[10.84,19.16]$ & & 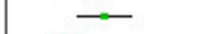 \\
\hline Wang 2012 & 31.56 & 3.23 & 10 & 22.52 & 5.62 & 9 & $9.2 \%$ & $9.04[4.86,13.22]$ & & - \\
\hline Zhang 2014 & 31.6 & 3.1 & 8 & 20.2 & 1.4 & 8 & $27.9 \%$ & $11.40[9.04,13.76]$ & & - \\
\hline Zhao 2009 & 35.8 & 13.2 & 10 & 22.7 & 7.6 & 10 & $1.8 \%$ & $13.10[3.66,22.54]$ & & \\
\hline Total $(95 \% \mathrm{Cl})$ & & & 49 & & & 48 & $100.0 \%$ & $11.60[10.32,12.88]$ & & $\bullet$ \\
\hline \multicolumn{9}{|c|}{$\begin{array}{l}\text { Heterogeneity: } \mathrm{Tau}^{2}=0.09 ; \mathrm{Chi}^{2}=4.14, \mathrm{df}=4(\mathrm{P}=0.39) ; \mathrm{I}^{2}=3 \% \\
\text { Test for overall effect: } Z=17.72(P<0.00001)\end{array}$} & $\begin{array}{ccc}-20 & -10 & 0 \\
\text { Favours [experimental] }\end{array}$ & 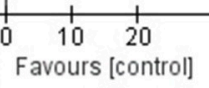 \\
\hline
\end{tabular}

\begin{tabular}{|c|c|c|c|c|c|c|c|c|c|c|}
\hline Guan 2010 & 930.7 & 131.5 & 6 & $1,285.8$ & 298.9 & 6 & $64.6 \%$ & $-1.42[-2.75,-0.09]$ & & \\
\hline Qu 2014 & 261.7 & 6.9 & 6 & 291.5 & 11.8 & 6 & $35.4 \%$ & $-2.85[-4.64,-1.05]$ & & \\
\hline \multirow{2}{*}{\multicolumn{9}{|c|}{$\begin{array}{l}\text { Heterogeneity: } \mathrm{Chi}^{2}=1.57, \mathrm{df}=1(\mathrm{P}=0.21) ; \mathrm{I}^{2}=36 \% \\
\text { Test for overall effect: } Z=3.54(P=0.0004)\end{array}$}} & & \\
\hline & & & & & & & & & $\begin{array}{cc} & 1 \\
-4 & -2 \\
\text { Favours [experimental] }\end{array}$ & 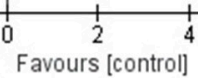 \\
\hline
\end{tabular}




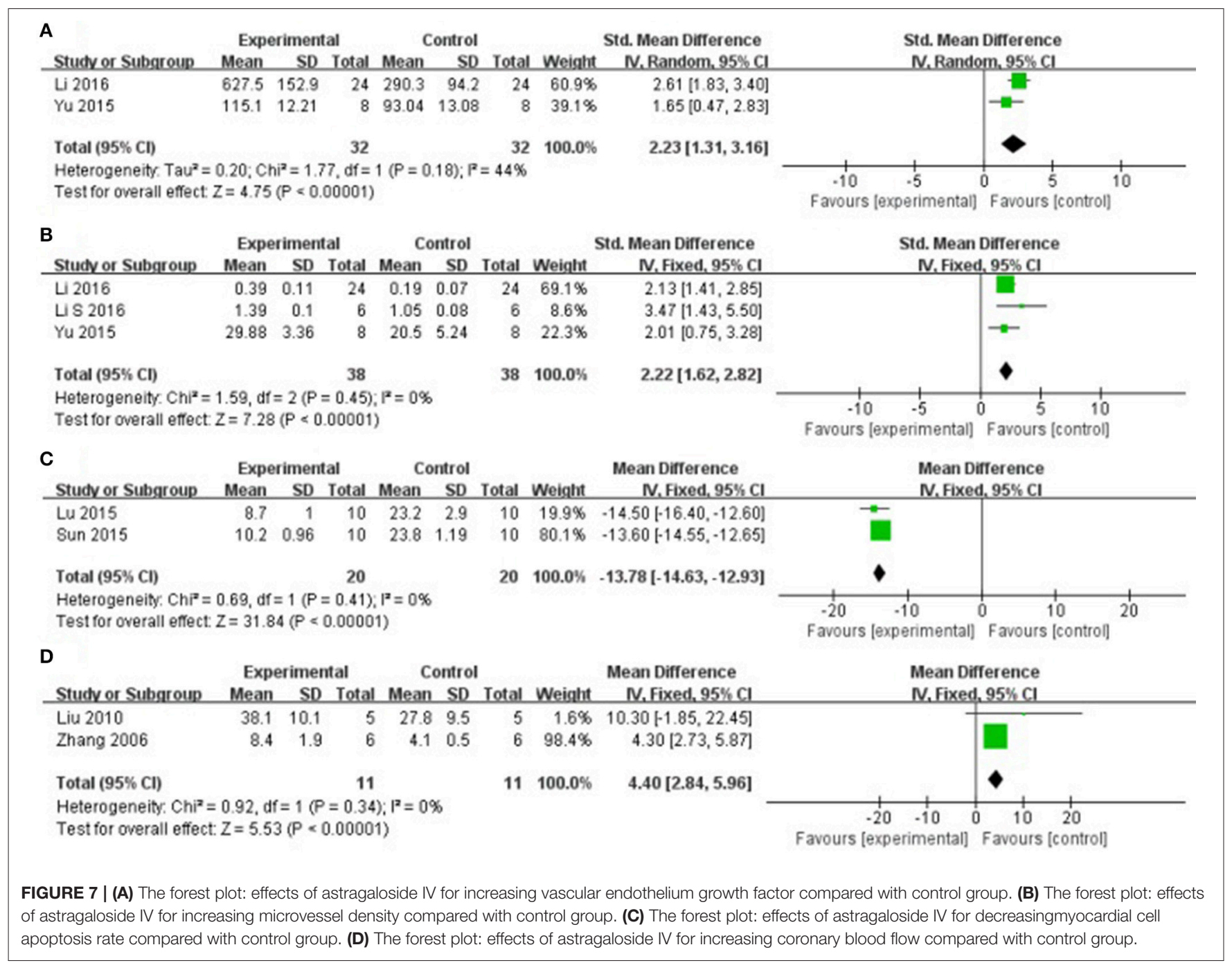

\section{DISCUSSION}

\section{Summary of Evidence}

In this preclinical systematic review, we assessed the efficacy of AS-IV for myocardial I/R injury according to 22 studies with 484 animals. The evidence available from present study revealed that AS-IV exerted potential cardioprotective function in acute MI largely through promoting angiogenesis, improvement of the circulation, antioxidant, anti-apoptosis and anti-inflammatory.

\section{Limitations}

First, no study provided calculation of sample size and blindness of model establishment and outcome measurement that is pesearch. Second, the deficiency of negative studies might lead the effecacy to be overestimated. Thus, the dominance of positive studies might lead the efficacy to be overestimated. Third, MI generally occurs in patients with other complications, such as old age, diabetes, hypertension, and hyperlipidemia (Blankstein et al., 2012); However, only 5 studies (Liu et al., 2010; Qu et al., 2014; Huang et al., 2015; Sun et al., 2015; Yu et al., 2015) selected appropriate animal model. Fourth, 3 studies (Liu et al., 2010; Gong and Sun, 2013; Li et al., 2016) adopted female animals, it cannot be ignored that the heart protection of estrogen has been reported both in clinical and preclinical studies (Menazza et al., 2017).

\section{Implications}

The high-quality preclinical studies are crucial to transform preclinical data to clinic (Ramirez et al., 2017). Thus, we suggest that further design of the studies should refer to the arrival guidelines (Carol et al., 2010) and use appropriate animals, random allocation, blinded induction of model, and blinded assessment of outcomes to improve the accuracy of the results.

The molecular and biological mechanisms of the cardioprorective effects of AS-IV have not been fully elucidated. The present study showed that AS-IV exerted the cardioprorection and the possible mechanisms are summarized as follows: (1) promoting angiogenesis and improving MVD (Yu et al., 2015; Li and Yang, 2016; Li et al., 2016) through increasing the expression of VEGF (Yu et al., 2015; Li et al., 2016) and basic 


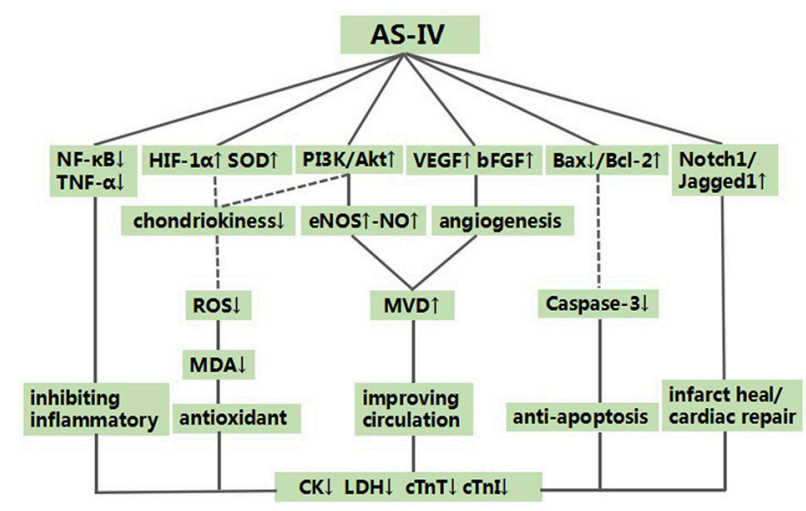

FIGURE 8 | A schematic representation of cardioprotective mechanisms of astragaloside IV for myocardial ischemia/reperfusion injury. Solid lines indicate established effects, whereas dashed lines represent putative mechanisms. fibroblast growth factor (bFGF) (Yu et al., 2015); (2) inhibition of apoptosis through down-regulating the expression of caspase-3 (Liu and Yi, 2014; Lu et al., 2015; Ma and Wang, 2015; Sun et al., 2015), and increasing the expression of $\mathrm{Bcl}-2$ and reducing the expression of Bax protein in the cardiac myocytes (Zhao et al., 2009; Tu et al., 2013; Liu and Yi, 2014; Lu et al., 2015; Ma and Wang, 2015; Cheng et al., 2016; Wang et al., 2018); (3) improvement of the coronary flow by enhancing the expression of NO via up-regulating the expression of endothelial nitric oxide synthase (eNOS) (Zhang et al., 2005; Liu et al., 2010); (4) upregulating HIF-1 $\alpha$ (Yu J. et al., 2017) and enhancing SOD-induced antioxidant via attenuating chondriokinesis to reduce the release of MDA (Zhang et al., 2005; Guan et al., 2010); otherwise, reducing the reactive oxygen species (ROS) to decrease myocardial cell lysis by regulating the PI3K/Akt/mTOR pathway (Zhang et al., 2014); (5) protecting against energy metabolism disorder through reducing the concentration of calcium in cardiac myocytes (Wang et al., 2012, 2018; Tu et al., 2013; Lu et al., 2015; Sun et al., 2015); (6) anti-inflammatory through inhibiting the expression of TNF- $\alpha$ (Lu et al., 2015) and NF-кB (Tu et al., 2013; Lu et al., 2015; Cheng et al., 2016); (7) upregulating Notch1/Jagged1 signaling (Yu J. et al., 2017) which may be involved in infarct healing and cardiac repair (Li et al., 2010; Gude and Sussman, 2012). As mentioned above, cardioprotective mechanism of AS-IV for myocardial I/R injury

\section{REFERENCES}

Baker, D., Lidster, K., Sottomayor, A., and Amor, S. (2014). Two years later: journals are not yet enforcing the ARRIVE guidelines on reporting standards for pre-clinical animal studies. PLoS Biol. 12:e1001756. doi: 10.1371/journal.pbio.1001756

Blankstein, R., Ahmed, W., Bamberg, F., Rogers, I. S., Schlett, C. L., Nasir, K., et al. (2012). Comparison of exercise treadmill testing with cardiac computed tomography angiography among patients presenting to the emergency room with chest pain: the Rule Out Myocardial Infarction Using Computer-Assisted Tomography (ROMICAT) was largely through promoting angiogenesis, improvement of the circulation, antioxidant, anti-apoptosis and anti-inflammatory.

It is well known that animal experiments have contributed to our understanding of effecacy and mechanisms for diseases (Hackam and Redelmeier, 2006). The present study showed ASIV significantly decreased the MI size and cardiac enzymes, decreased cardiac troponin and increased the decline degree in ST segment. Therefore, it provides a preclinical evidencebased approach to develop AS-IV for acute MI. However, the translation of preclinical experiment which results in a prediction of the effectiveness of treatment strategies in clinical trials is still challenging (Hackam, 2007). The application of excessive drug doses and the timing of drug administration in animal models, which are inapplicable for human disease, are considered to be two of the main reasons for the failure to translate from animal models to human (Baker et al., 2014). In the present study, doses of AS-IV and timing for initial administration in animal models were inconsistent among the 22 included studies. Thus, we suggest further studies to determinate the optimal gradient doses and timing of administration in animal models of myocardial I/R injury. After that, given the huge gap between the animal studies and the clinical trials, the rigorous RCTs of AS-IV are needed.

\section{CONCLUSION}

We have provided a first comprehensive systematic review of AS-IV on animal studies and the findings indicate that AS-IV exerted potential cardioprotective function in acute myocardial I/R injury largely through promoting angiogenesis, improvement of the circulation, antioxidant, anti-apoptosis and anti-inflammatory.

\section{AUTHOR CONTRIBUTIONS}

QZ, J-ZZ, X-YB, P-CZ, QT, Y-YH, Q-HZ, and K-JZ designed the study. QZ and J-ZZ collected the data. G-QZ and J-ZZ performed all analyses. QZ, G-QZ, and YW wrote the manuscript. All authors contributed to writing of this manuscript.

\section{ACKNOWLEDGMENTS}

This project was supported by the grant of National Natural Science Foundation of China (81573750/81473491/81173395/H2902). study. Circulation 5, 233-242. doi: 10.1161/CIRCIMAGING.111.9 69568

Carol, K., Willian, J. B., Innes, C. C., Micheal, E., and Douglas, G. A. (2010). Improving bioscience research reporting: the ARRIVE guidelines for reporting animal research. PLoS Biol. 8:e1000412. doi: 10.1371/journal.pbio. 1000412

Cheng, S., Yu, P., Yang, L., Shi, H., He, A., Chen, H., et al. (2016). Astragaloside IV enhances cardioprotection of remote ischemic conditioning after acute myocardial infarction in rats. Am. J. Transl. Res. 8, 4657-4669.

Cui, D. M., Chen, Y. J., Liu, D. H., Meng, K. Z., and Zhu, H. S. (2013). Effect of astragalus sapson on hemodynamics and neuroendocrine of rats 
with heart failure following myocardial infarction. J. Navy Med. 34, 18-20. doi: 10.3969/j.issn.1009-0754.2013.01.008

Damman, P., Van't, H. A., Ten Berg, J., Jukema, J. W., Appelman,. Y., Liem, A. H., et al. (2015). 2015 ESC guidelines for the management of acute coronary syndromes in patients presenting without persistent ST-segment elevation: comments from the Dutch ACS working group. Netherlands Heart J. 25, 181-185. doi: 10.1007/s12471-016-0 939-y

Dariush, M., Emelia, J. B., Alan, S. G., Donna, K. A., Michael, J. B., Mary, C., et al. (2016). Heart disease and stroke statistics-2016 update: a report from the american heart association. Circulation 133, e38-360. doi: $10.1161 /$ CIR.0000000000000350

Gong, A. D., and Sun, J. H. (2013). The influence of Astragaloside IV on myocardial ischemia and arrhythmia in rat model myocardial $\mathrm{Ca} 2+$ concentration and its anti-arrhythmia mechanism. Asia Pacific Trad. Med. 9, 11-12. doi: 10.3969/j.issn.1673-2197.2013.08.006

Guan, F. Y., Li, H., and Yang, S. J. (2010). Effect of astragaloside IV on myocardial ischemia-reperfusion injury in rats via $\mathrm{NO}$ production and PKCE promotion. J. Jilin Univ. (Med. Edn). 36, 340-344. doi: 10.13481/j.1671-587x.2010.02.014

Gude, N., and Sussman, M. (2012). Notch signaling and cardiac repair. J. Mol. Cell. Cardiol. 52, 1226-1232. doi: 10.1016/j.yjmcc.2012.03.007

Hackam, D. G. (2007).Translating animal research into clinical beneft. Br. Med. J. 334, 163-164. doi: 10.1136/bmj.39104.362951.80

Hackam, D. G., and Redelmeier, D. A. (2006). Translation of research evidence from animals to humans. JAMA 296, 1731-1732. doi: 10.1001/jama.296.14.1731

He, Y.-G., Li, W.-F., Yi, H.-L., Zheng, H., and Xi, J.-K. (2014). Mitochondrialmechanism of Astragaloside IV induced inhibition of GSK-3 $\beta$ in myocardial ischemia/reperfusion injury in rats. Chin. Pharmacol. Bull. 30, 402-406. doi: 10.3969/j.issn.1001-1978.2014.0 3.023

Huang, L., Wang, D. W., Yan, X., and Huang, W. X. (2015). Regulatory effects of astragaloside on myocardial injury and cell autophagy induced by ischemia reperfusion in rats. Chin. J. Integr. Med. Cardio/Cerebrovasc. Dis. 13, 752-754. doi: $10.3969 /$ j.issn.16721349.2015.06.016

Li, J. C., Si, J. W., Liu, H. T., Zhu, F. H., Wang, W., Ma, J., et al. (2016). Effect of Astragaloside IV on angiogenesis maturity in mice with myocardial infaction. Chin. Heart J. 29, 269-274. doi: 10.13191/j.chj.2017.0070

Li, L., Hou, X., Xu, R., Liu, C., and Tu, M. (2017). Research review on the pharmacological effects of astragaloside IV. Fundam. Clin. Pharmacol. 31, 17-36. doi: 10.1111/fcp. 12232

Li, S., and Yang, D. (2016). Astragaloside IV Improves Cardiac Function Via Angiogenesis in Mice After Myocardial Infaction. Master Thesis. Available online at: http://kns.cnki.net/kns/brief/default_result.aspx (Accessed May, 2016).

Li, Y., Hiroi, Y., and Liao, J. K. (2010). Notch signaling as an important mediator of cardiac repair and regeneration after myocardial infarction. Trends Cardiovasc. Med. 20, 228-231. doi: 10.1016/j.tcm.2011.1 1.006

Liu, H., and Yi, D.-H. (2014). The Effects of Astragaloside IV Post-treatment Against Myocardial Ischemia and Reperfusion by Activation of the Hif- $1 \alpha$ Signalingpathway. Master Thesis. Available online at: http://d.wanfangdata. com.cn/Thesis/D527921 (Accessed November, 2014).

Liu, J., Ding, Y. J., Lin, N., and Shu, B. (2010). Protective effect of astragaloside on cardiac function injury induced by myocardial ischemia in beagles. China Practical Med. 5, 12-15. doi: 10.3969/j.issn.1673-7555.2010.3 3.006

Lu, M. L., Tang, F. T., Zhang, J., Luan, A. N., Mei, M., Xu, C. H., et al. (2015). Astragaloside IV attenuates injury caused by myocardial ischemia/reperfusion in rats via regulation of toll-like receptor 4/Nuclear Factor- $\kappa \mathrm{B}$ signaling pathway. Phytother. Res. 4, 599-606. doi: 10.1002/ptr.5297

Luo, K. Q., Long, H. B., and Xu, B. C. (2015). Reduced apoptosis afteracutemyocardial infarction by simvastatin. Cell Biochem. Biophys. 71, 735-740. doi: 10.1007/s12013-014-0257-1

Ma, X. L., and Wang, G. M. (2015). Protective effect of astragaloside IV on myocardial ischemia-reperfusion injury in rats pretreated with astragaloside IV by gavage. Chin. J. Gerontol. 35, 4795-4796. doi: 10.3969/j.issn.1005-9202.2015.17.026
Malcolm, R. M.,Tori, O., David, W. H., and Geoffrey, A. D. (2004). Pooling of animal experimental data reveals influence of study design and publication bias.Stroke 35, 1203-1208. doi: 10.1161/01.STR.0000125719.25 853.20

Maria, S., James, A. C., Daniel, C. S., and Paul, A. I. (2016). Featured Article: pharmacological postconditioning with delta opioid attenuates myocardial reperfusion injury in isolated porcine hearts. Exp. Biol. Med. 242, 986-995. doi: $10.1177 / 1535370216684041$

Menazza, S., Sun, J., Appachi, S., Chambliss, K. L., Kim, S. H., Aponte, A., et al. (2017). Non-nuclear estrogen receptor alpha activation in endothelium reduces cardiac ischemia-reperfusion injury in mice. J. Mol. Cell. Cardiol. 107, 41-51. doi: 10.1016/j.yjmcc.2017.04.004

Qu, Y. C., Wang, D. W., Yan, X., Huang, X. W., and Wang, J. (2014). Astragaloside IV reduces myocardial injury induced by ischemia-reperfusion in rats. Chin. J. Gerontol. 34, 3404-3405. doi: 10.3969/j.issn.1005-9202.2014.12.090

Ramirez, F. D., Motazedian, P., Jung, R. G., Santo, P. D., MacDonald, Z. D., Moreland, R., et al. (2017). Methodological rigor in preclinical cardiovascular studies targets to enhance reproducibility and promote research translation. Circ. Res. 120, 1916-1926. doi: 10.1161/CIRCRESAHA.117.310628

Ren, S., Zhang, H., Mu, Y., Sun, M., and Liu, P. (2013). Pharmacological effects of AstragalosideIV: a literature review. J. Tradit. Chin. Med. 33, 413-416. doi: 10.1016/S0254-6272(13)60189-2

Richard, K. (2011). Primary prevention of coronary heart disease: integration of new data, evolving views, revised goals, and role of rosuvastatin in management. A comprehensive survey. Drug Design Dev. Ther. 5, 325-380. doi: 10.2147/DDDT.S14934

Rob, B. M., Kimberley, E. W., Marc, T. A., Martin, L. S., Emily, S. S., and Marlies, L. (2014). The usefulness of systematic reviews of animal experiments for the design of preclinical and clinical studies. ILAR J. 55, 427-437. doi: 10.1093/ilar/ilu043

Schmidt, M. R., Smerup, M., Konstantinov, I. E., Shimizu, M., Li, J., Cheung, M., et al. (2007). Intermittent peripheral tissue ischemia during coronary ischemia reduces myocardial infarction through a KATP-dependent mechanism: first demonstration of remote ischemic perconditioning. Am. J. Physiol. Heart Circ. Physiol. 292, 1883-1890. doi: 10.1152/ajpheart.00617

Stewart, L. A., Clarke, M., Rovers, M., Riley, R. D., Simmonds, M., Stewart, G., et al. (2015). Preferred reporting items for systematic review and metaanalyses of individual participant data: the PRISMA-IPD Statement. JAMA 313, 1657-1665. doi: 10.1001/jama.2015.3656

Sun, Y. L., Xin, Q. F., Li,. C. Y., and Wang, N. (2015). Effect of Astragaloside IV decreases non-infarction zone cell apoptosis in aged rats with myocardial infarction by inhibiting endoplasmic reticulum stress-related apoptosis. Pharmacol. Clin. Chin. Mat. Med. 31, 29-32. doi: 10.13412/j.cnki.zyyl.2015.01.010

Tu, L., Pan, C. S., Wei, X. H., Li, Y., Liu, Y., and Fan, J. Y., et al. (2013). Astragaloside IV protects heart from ischemia and reperfusion injury via energy regulation mechanisms. Microcircultion 20, 736-747. doi: 10.1111/micc.12074

Wang, J., Gao, D. D., Wang, W. H., Dai, J. Y., Yang, Y., and Hou, X. W. (2018). Calcium-sensing receptor contributes to the protective effect of astragaloside IV on myocardial ischemia reperfusion injury. J. Shenyang Pharmaceut. Univ. 35, 312-318. doi: 10.14066/j.cnki.cn21-1349/r.2018.0 4.011

Wang, Y., Ji, Y. S., Xing, Y. F., Li, X., and Gao, X. M. (2012). Astragalosides rescue both cardiac function and sarcoplasmic reticulum $\mathrm{Ca} 2+$ transport in rats with chronic heart failure. Phytother. Res. Ptr. 26, 231-238. doi: 10.1002/ ptr.3492

Yellon, D. M., and Hausenloy, D. J. (2007). Myocardial reperfusion injury. N. Engl. J. Med. 357, 1121-1135. doi: 10.1056/NEJMra071667

Yu, J. M., Zhang, X. B., Jiang, W., Wang, H. D., and Zhang, Y. N. (2015) Astragalosides promote angiogenesis via vascular endothelial growth factor and basic fbroblast growth factor in a rat model of myocardial infarction. Mol. Med. Rep. 12, 6718-6726. doi: 10.3892/mmr.2015.4307

Yu, J., Zhang, X., and Zhang, Y. (2017). Astragaloside attenuates myocardial injury in a rat model of acute myocardial infarction by upregulating hypoxia inducible factor- $1 \alpha$ and Notch1/Jagged1 signaling. Mol. Med. Rep. 15, 4015-4020. doi: $10.3892 / \mathrm{mmr} .2017 .6522$ 
Yu, L. J., Zhang, K. J., Zhu, J. Z., Zheng, Q., Bao, X. Y., Wang, Y., et al. (2017). Salvianolic acid exerts cardioprotection through promoting: angiogenesis in animal models of acute myocardial infarction: preclinical evidence. Oxid. Med. Cell. long. 2017:8192383. doi: 10.1155/2017/81 92383

Zhang, J., Ma, C. L., and Wang, G. Z. (2014). Improvement effects of astragaloside IV on myocardial focal ischemia-reperfusion injury and its influence in PI3K/Akt/mTOR signaling pathway. $J$. Jilin Univ. (Med. Ed). 40, 991-996. doi: 10.13481/j.1671-587x.201 40517

Zhang, W. D., Chen, D., Zhang, C., Liu, R. H., Li, H. L., and Chen, H. Z., et al. (2005). Astragaloside IV from Astragalus membranaceus shows cardioprotection during myocardial ischemia in vivo and in vitro. Planta Med. 72, 4-8. doi: 10.1055/s-2005-8 73126
Zhao, Z. Y., Wang, W. T., Wang, F., Zhao, K. R., Han, Y. M., Xu, W. R., et al. (2009). Effects of Astragaloside IV on heart failure in rats. Chin. Med. 4, 1-6. doi: 10.1186/1749-8546-4-6

Conflict of Interest Statement: The authors declare that the research was conducted in the absence of any commercial or financial relationships that could be construed as a potential conflict of interest.

Copyright $\odot 2018$ Zheng, Zhu, Bao, Zhu, Tong, Huang, Zhang, Zhang, Zheng and Wang. This is an open-access article distributed under the terms of the Creative Commons Attribution License (CC BY). The use, distribution or reproduction in other forums is permitted, provided the original author(s) and the copyright owner(s) are credited and that the original publication in this journal is cited, in accordance with accepted academic practice. No use, distribution or reproduction is permitted which does not comply with these terms. 